\title{
Distant Metastasis from Benign Solitary Fibrous Tumor of the Kidney
}

\author{
Hideo Sasaki ${ }^{a}$ Tsuyoshi Kurihara ${ }^{a}$ Yuichi Katsuoka ${ }^{a}$ Toru Nakano ${ }^{a}$ \\ Maki Yoshioka $^{a}$ Satetsu Miyano $^{a}$ Yuichi Sato $^{a}$ Iwao Uejima $^{\text {b }}$ \\ Masahiro Hoshikawa ${ }^{c}$ Masayuki Takagi ${ }^{c}$ Tatsuya Chikaraishi ${ }^{a}$ \\ Departments of ${ }^{a}$ Urology, ${ }^{b}$ Radiology and ${ }^{C}$ Pathology, St. Marianna University School of \\ Medicine, Kawasaki, Japan
}

\section{Key Words}

Solitary fibrous tumor $\cdot$ Metastasis $\cdot$ Kidney $\cdot$ Malignant $\cdot$ CD34

\begin{abstract}
Solitary fibrous tumor (SFT) rarely occurs in the kidneys, and only one reported case of renal SFT has shown distant metastasis. We report the second case of renal SFT exhibiting distant metastasis. A 48-year-old man was referred to our hospital because of a right renal mass. An abdominal CT scan detected a large renal tumor, which was suspected to be a renal cell carcinoma. Right radical nephrectomy was performed, and the tumor was found to measure $28 \times 18 \times 10 \mathrm{~cm}$. The pathological diagnosis was benign solitary fibrous tumor of the kidney. Eight years after the operation, lung and liver metastases developed. Pulmonary segmentectomy and partial hepatectomy were performed. The pathological diagnoses of these resected tissue specimens were compatible with benign SFT.
\end{abstract}

\section{Introduction}

Solitary fibrous tumor (SFT) was first reported by Klemperer and Rabin in 1931 as a tumor of the pleura [1]. SFT is a rare type of spindle cell neoplasm that usually arises in the pleura [2]. However, it has also been reported to occur at other sites. SFT of the kidneys is rare, and furthermore, distant metastasis from SFT is extremely rare. We report the second case of renal SFT to exhibit distant metastasis. 


\section{Case Report}

A 48-year-old man was referred to our hospital because of a right renal mass. A physical examination revealed a hard right abdominal mass, and a subsequent CT scan detected a large right renal tumor, which was suspected to be a renal cell carcinoma (fig. 1). The patient underwent radical right nephrectomy. In gross appearance, the tumor measured $28 \times 18 \times$ $10 \mathrm{~cm}$, and displayed cystic changes, necrosis and hemorrhage with grayish-white cut surfaces. The tumor developed from the upper pole of the kidney adjacent to the renal capsule and markedly compressed the normal kidney into the lower side.

Microscopically, the tumor was found adjacent to the renal capsule; but the site of origin was ambiguous. It consisted of spindle-shaped cells with scant cytoplasm accompanied by prominent hyalinized collagenous tissue, which displayed hemangiopericytomatous patterns. The cells did not display cytological atypia, and no mitotic figures were detected. Immunohistochemical staining was positive for CD34, vimentin, and CD99 and negative for keratin, EMA, CD10, CD31, factor VIII, $\alpha$ SMA, Bcl-2, S-100, and CD117 (fig. 2). These findings resulted in a diagnosis of benign SFT.

However, 8 years after the original operation, follow-up CT detected a lung nodule and multiple liver nodules (fig. 3), which were consistent with metastasis from the primary renal SFT. Ultrasound-guided liver needle biopsy was performed and led to a pathological diagnosis of SFT. Surgical resection was planned to treat the tumors, and partial hepatectomy was performed. Two months after the partial hepatectomy, pulmonary segmentectomy was performed. Microscopically, the metastatic tumors were composed of spindle cells in a collagenous stroma containing hemangiopericytomatous structures. The cells did not display cytological atypia, and no mitotic figures were detected. Although immunohistochemical staining for CD34 was negative in the resected tissue from the liver and weakly positive in the resected tissue from the lungs, the specimens were positively stained for vimentin and CD99 and negative for CD10, factor VIII, $\alpha$ SMA, Bcl-2, and S-100 (fig. 2). The pathological diagnosis was benign SFT.

As the CD34 expression of the primary tumor and metastatic lesion differed, we reevaluated the pathological findings of the primary renal SFT. Immunohistochemically, we observed CD34 labeling in 55\% of the tumor cells with no expression in the remaining component. The CD34-negative section of the lesion was morphologically indistinguishable from the CD34-positive part of the lesion. Pathologically malignant findings were not observed in any of the lesions. At 12 months after surgery, the patient is healthy and has not displayed any evidence of recurrence or metastasis.

\section{Discussion}

SFT was first reported by Klemperer and Rabin in 1931 as a tumor of the pleura [1]. SFT is a rare spindle cell neoplasm that usually arises in the pleura [2]. However, in recent years, there have been several reports of SFT arising in other organs, including the kidneys [2,3]. The histogenesis of SFT has been debated for years, but recent studies have indicated that it has a mesenchymal origin [4]. Immunohistochemical studies are useful for establishing a diagnosis, especially staining for CD34, which is considered to be a marker of SFT [5]. In addition, most SFT are diffusely positive for Bcl-2 and CD99 [6]. Loss of Bcl-2 was closely related to high malignant potential in extrathoracic SFT [7]. Surgical resection has been demonstrated to be beneficial in the treatment of SFT. Even if the SFT is histologically 
diagnosed as malignant, complete excision of the tumor is associated with a favorable prognosis [8].

SFT of the kidneys is rare, and only 67 cases have been reported (table 1). Most of these tumors were preoperatively diagnosed as renal cell carcinoma, and radical nephrectomy was the standard treatment. Pathologically, 61 tumors were diagnosed as benign and 6 tumors were diagnosed as malignant. All tumors except one displayed a favorable prognosis, with no evidence of recurrence during the follow-up period, which ranged from 2 to 89 months. Immunohistochemically, most tumors were positive for CD34. Although the origin of renal SFT is difficult to determine, some reported renal SFT originated from the renal capsule [3], and Yamada et al. [9] speculated that renal SFT originates from primitive mesenchymal cells located in the renal capsule. Further research is necessary to clarify the origin of renal SFT.

Fine et al. [10] reported the first case of malignant renal SFT to develop distant metastasis. Their case involved a 76-year-old man who was treated with left radical nephrectomy. Pathologically, $10 \%$ of the renal tumor consisted of typical benign SFT; however, the remaining component was composed of pleomorphic, spindle-shaped sarcoma cells with frequent mitoses and necrotic foci. Immunohistochemically, CD34 labeling was observed in the benign SFT component with no CD34 expression in the sarcomatous component. Four months after surgery, multiple lung metastases developed. This was the first reported case of malignant renal SFT involving distant metastasis. In this patient, neither metastasectomy nor a histological examination of the metastatic lesion was performed.

To the best of our knowledge, our case is the second reported case of renal SFT to involve distant metastasis and is the first reported case of renal SFT to include the pathological findings of the metastatic lesion. Furthermore, the primary tumor and the resected tissue from the metastatic site were pathologically benign, and no malignant findings were observed in any of the lesions. Immunohistochemically, 55\% of the primary tumor displayed positive CD34 labeling, whereas no CD34 expression was detected in the remaining component. The CD34-negative part of the lesion was morphologically indistinguishable from its CD34-positive region. In addition, CD34 expression was negative in the resected tissue from the liver and weakly positive in the resected tissue from the lungs. Thus, we postulated that the loss of CD34 expression might promote tumor metastasis to other organs, and could lead to malignant transformation from the benign tumor relevant to fatal outcome [11]. Moreover, our case of SFT was negative in Bcl-2 expression in the primary tumor and metastatic lesions; this may also participate in malignant outcome [7]. Further research is needed to clarify these points.

Our case is very similar to that reported by Hasegawa et al. [12]. They described an extrathoracic SFT that metastasized to the lungs. Neither the primary nor metastatic lesions displayed any atypical features. Thus, extrathoracic SFT might have the potential to recur or metastasize, even in the absence of atypical pathological features [12]. Renal SFT is generally reported to be a benign tumor; however, the follow-up periods in the 67 reported cases might not have been sufficient to allow the clinical outcome to be fully evaluated (table 1). A longer follow-up period might be necessary to definitively evaluate the clinical outcome of renal SFT.

\section{Disclosure Statement}

The authors have no conflicts of interest to disclose. 


\begin{tabular}{l|l}
\hline Case Rep Nephrol Urol 2013;3:1-8 & \\
\hline DOI: $10.1159 / 000346850$ & $\begin{array}{l}\text { ○ 2013 S. Karger AG, Basel } \\
\text { www.karger.com/cru }\end{array}$ \\
\hline
\end{tabular}

Sasaki et al.: Distant Metastasis from Benign Solitary Fibrous Tumor of the Kidney

\section{References}

1 Klemperer P, Rabin CB: Primary neoplasm of the pleura: a report of five cases. Arch Pathol 1931;11:385412.

2 Goodlad JR, Fletcher CD: Solitary fibrous tumour arising at unusual sites: analysis of a series. Histopathology 1991;19:515-522.

-3 Gelb AB, Simmons ML, Weidner N: Solitary fibrous tumor involving the renal capsule. Am J Surg Pathol 1996;20:1288-1295.

4 Battiffora H, McCaughey WTE: Tumors of the serosal membranes. Atlas of Tumor Pathology, 3rd, fascicle. Washington, DC: Armed Forces Institution of Pathology, 1995.

-5 Bortolotti U, Calabrò F, Loy M, Fasoli G, Altavilla G, Marchese D: Giant intrapericardial solitary fibrous tumor. Ann Thorac Surg 1992;54:1219-1220.

-6 Magro G, Emmanuele C, Lopes M, Vallone G, Greco P: Solitary fibrous tumour of the kidney with sarcomatous overgrowth. Case report and review of the literature. APMIS 2008;116:1020-1025.

7 Takizawa I, Saito T, Kitamura Y, Arai K, Kawaguchi M, Takahashi K, Hara N: Primary solitary fibrous tumor (SFT) in the retroperitoneum. Urol Oncol 2008;26:254-259.

8 Ito H, Fukuda M, Imamura Y, Fuse H: A malignant solitary fibrous tumor in the retroperitoneum. Int J Clin Oncol 2008;13:173-175.

-9 Yamada H, Tsuzuki T, Yokoi K, Kobayashi H: Solitary fibrous tumor of the kidney originating from the renal capsule and fed by the renal capsular artery. Pathol Int 2004;54:914-917.

10 Fine SW, McCarthy DM, Chan TY, Epstein JI, Argani P: Malignant solitary fibrous tumor of the kidney: report of a case and comprehensive review of the literature. Arch Pathol Lab Med 2006;130:857-861.

11 Yokoi T, Tsuzuki T, Yatabe Y, Suzuki M, Kurumaya H, Koshikawa T, Kuhara H, Kuroda M, Nakamura N, Nakatani Y, Kakudo K: Solitary fibrous tumour: significance of p53 and CD34 immunoreactivity in its malignant transformation. 1998;32:423-432.

12 Hasegawa T, Matsuno Y, Shimoda T, Hasegawa F, Sano T, Hirohashi S: Extrathoracic solitary fibrous tumors: their histological variability and potentially aggressive behavior. Hum Pathol 1999;30:1464-1473. 
Table 1. Clinicopathological findings of renal solitary fibrous tumors in the literature

\begin{tabular}{|c|c|c|c|c|c|c|c|c|c|c|c|c|c|}
\hline Case & Year & $\begin{array}{l}\text { Age } \\
\text { years }\end{array}$ & Sex & Symptom & Side & $\begin{array}{l}\text { Affected } \\
\text { site }\end{array}$ & $\begin{array}{l}\text { Tumor } \\
\text { size, } \mathrm{cm}\end{array}$ & Treatment & Histology & $\begin{array}{l}\text { Follow- } \\
\text { up }\end{array}$ & Outcome & CD34* & Authors and journals \\
\hline 1 & 1996 & 48 & M & $\begin{array}{l}\text { Back pain and } \\
\text { macrohematuria }\end{array}$ & $\mathrm{R}$ & $\begin{array}{l}\text { Renal } \\
\text { capsule }\end{array}$ & 3 & Nephrectomy & BEN & 0.1 & DNOD & POS & $\begin{array}{l}\text { Gelb et al., Am J Surg Pathol } \\
20: 1288\end{array}$ \\
\hline 2 & 1996 & 45 & F & Incidental & $\mathrm{R}$ & Kidney & 6 & Nephrectomy & BEN & 8 & NED & $\operatorname{POS}(2 / 3)$ & Fain et al., J Urol Pathol 4:227 \\
\hline 3 & 1996 & 46 & $\mathrm{~F}$ & Incidental & $\mathrm{R}$ & Kidney & 7.2 & Nephrectomy & BEN & 33 & NED & $\operatorname{POS}(2 / 3)$ & Fain et al., J Urol Pathol 4:227 \\
\hline 4 & 1996 & 51 & M & Incidental & $\mathrm{L}$ & Kidney & 4.5 & Nephrectomy & BEN & 2 & NED & POS (2/3) & Fain et al., J Urol Pathol 4:227 \\
\hline 5 & 1997 & 33 & $\mathrm{~F}$ & Abdominal pain & $\mathrm{R}$ & Peripelvis & 3.5 & Nephrectomy & BEN & 89 & NED & POS & $\begin{array}{l}\text { Fukunaga et al., Hispathology } \\
30: 451\end{array}$ \\
\hline 6 & 1997 & 36 & $\mathrm{~F}$ & Abdominal pain & $\mathrm{L}$ & Peripelvis & 2 & Nephrectomy & BEN & 12 & NED & POS & $\begin{array}{l}\text { Fukunaga et al., Hispathology } \\
30: 451\end{array}$ \\
\hline 7 & 1998 & 59 & M & Incidental & $\mathrm{L}$ & $\begin{array}{l}\text { Renal } \\
\text { capsule }\end{array}$ & NA & Nephrectomy & BEN & NA & NA & POS & $\begin{array}{l}\text { Ookouci S et al., Jpn J Radiol } \\
\text { 58: } 539\end{array}$ \\
\hline 8 & 1998 & 57 & $\mathrm{M}$ & Incidental & $\mathrm{L}$ & Kidney & 7 & Tumorectomy & BEN & NA & NA & POS & $\begin{array}{l}\text { Tanahashi C et al., Proc Jpn Soc } \\
\text { Pathol 87:510 }\end{array}$ \\
\hline 9 & 1999 & 64 & M & Macrohematuria & $\mathrm{R}$ & Kidney & 4.5 & Nephrectomy & BEN & 8 & NED & POS & $\begin{array}{l}\text { Hasegawa et al., Hum Pathol } \\
30: 1464\end{array}$ \\
\hline 10 & 1999 & 71 & $\mathrm{~F}$ & Incidental & $\mathrm{L}$ & Kidney & 9 & Nephrectomy & BEN & NA & NA & NA & $\begin{array}{l}\text { Kojima K et al., Jap-Deu Med } \\
\text { Beriche 44:185 }\end{array}$ \\
\hline 11 & 2000 & 66 & $\mathrm{~F}$ & $\begin{array}{l}\text { Abdominal pain and } \\
\text { macrohematuria }\end{array}$ & $\mathrm{R}$ & Kidney & 9 & Nephrectomy & BEN & 9 & NED & POS & Leroy et al., Urol Int 65:49 \\
\hline 12 & 2000 & 72 & F & NA & $\mathrm{L}$ & Kidney & 8 & Nephrectomy & BEN & 10 & NED & POS & $\begin{array}{l}\text { Morimitsu et al., APMIS } \\
108: 617\end{array}$ \\
\hline 13 & 2000 & 56 & F & Incidental & $\mathrm{L}$ & $\begin{array}{l}\text { Renal } \\
\text { capsule }\end{array}$ & 5 & Tumor resection & BEN & NA & NA & NA & $\begin{array}{l}\text { Ikeda A et al., J Hiroshima Med } \\
\text { Assoc 53:640 }\end{array}$ \\
\hline 14 & 2001 & 70 & M & Incidental & $\mathrm{R}$ & $\begin{array}{l}\text { Renal } \\
\text { pelvis }\end{array}$ & 6 & Nephrectomy & BEN & 60 & NED & POS & Yazaki et al., Int J Urol 8:504 \\
\hline 15 & 2001 & 28 & $\mathrm{~F}$ & Abdominal pain & $\mathrm{L}$ & Kidney & 15 & Nephrectomy & BEN & 12 & NED & POS & $\begin{array}{l}\text { Cortes-Gutierrez et al., J Urol } \\
166: 60\end{array}$ \\
\hline 16 & 2001 & 41 & $\mathrm{M}$ & Macrohematuria & $\mathrm{L}$ & Kidney & 14 & Nephrectomy & BEN & 48 & NED & POS & $\begin{array}{l}\text { Wang J et al., Am J Surg Pathol } \\
25: 1194\end{array}$ \\
\hline 17 & 2001 & 72 & M & $\begin{array}{l}\text { Abdominal } \\
\text { discomfort }\end{array}$ & $\mathrm{R}$ & Kidney & 13 & Nephrectomy & BEN & 5 & NED & POS & $\begin{array}{l}\text { Wang J et al., Am J Surg Pathol } \\
25: 1194\end{array}$ \\
\hline 18 & 2002 & 57 & $\mathrm{M}$ & Incidental & $\mathrm{L}$ & Kidney & 6 & Nephrectomy & BEN & NA & NA & POS & $\begin{array}{l}\text { Miyazaki N et al., Jpn Red } \\
\text { Cross Med J 54:182 }\end{array}$ \\
\hline 19 & 2002 & 58 & $\mathrm{M}$ & Incidental & $\mathrm{L}$ & Kidney & NA & Nephrectomy & BEN & 9 & NED & NA & $\begin{array}{l}\text { Inokawa E, J Hiroshima Med } \\
\text { Assoc 55:1057 }\end{array}$ \\
\hline 20 & 2002 & 31 & F & Flank pain & $\mathrm{R}$ & Kidney & 8.6 & Nephrectomy & BEN & 8 & NED & POS & $\begin{array}{l}\text { Magro G, Pathol Res Pract } \\
198: 37\end{array}$ \\
\hline 21 & 2003 & 64 & $\mathrm{~F}$ & Microhematuria & $\mathrm{R}$ & Kidney & 4 & Nephrectomy & BEN & 7 & NED & POS & $\begin{array}{l}\text { Li S et al., Hinyokika Kiyo } \\
49: 121\end{array}$ \\
\hline 22 & 2003 & 51 & $\mathrm{~F}$ & NA & $\mathrm{R} / \mathrm{L}$ & Kidney & $25 \& 2$ & Tumor resection & BEN & NA & NA & NA & $\begin{array}{l}\text { Llarena Ibarguren et al., Arch } \\
\text { Esp Urol 56:835 }\end{array}$ \\
\hline 23 & 2003 & 35 & $\mathrm{M}$ & NA & $\mathrm{R}$ & Kidney & 17 & Nephrectomy & BEN & 6 & NED & NA & $\begin{array}{l}\text { Durand X et al., Prog Urol } \\
\text { 13:491 }\end{array}$ \\
\hline 24 & 2003 & 60 & $\mathrm{~F}$ & NA & $\mathrm{R}$ & Kidney & 11 & Nephrectomy & BEN & 48 & NED & NA & $\begin{array}{l}\text { Bugel H et al., Prog Urol } \\
\text { 13:1397 }\end{array}$ \\
\hline 25 & 2004 & 67 & $\mathrm{M}$ & Incidental & $\mathrm{L}$ & Kidney & 4.5 & Tumorectomy & BEN & 5 & NED & POS & $\begin{array}{l}\text { Toriyama S et al., Hinyokika } \\
\text { Kiyo 50:138 }\end{array}$ \\
\hline 26 & 2004 & 83 & $\mathrm{M}$ & NA & $\mathrm{R}$ & Kidney & 9 & Nephrectomy & BEN & 18 & NED & POS & Gres P et al., Prog Urol 14:65 \\
\hline 27 & 2004 & 53 & M & $\begin{array}{l}\text { Flank pain and } \\
\text { swelling }\end{array}$ & $\mathrm{R}$ & $\begin{array}{l}\text { Renal } \\
\text { capsule }\end{array}$ & 14 & Tumor resection & BEN & 36 & DNOD & POS & $\begin{array}{l}\text { Kunieda K et al., Surg Today } \\
34: 90\end{array}$ \\
\hline 28 & 2004 & 59 & M & Incidental & $\mathrm{L}$ & $\begin{array}{l}\text { Renal } \\
\text { capsule }\end{array}$ & 6.8 & Nephrectomy & BEN & 48 & NED & POS & $\begin{array}{l}\text { Yamada H et al., Pathol Int } \\
54: 914\end{array}$ \\
\hline $\begin{array}{l}29- \\
35\end{array}$ & 2005 & $29-79$ & NA & $\begin{array}{l}5 \text { incidental and } 2 \\
\text { flank pain }\end{array}$ & NA & $\begin{array}{l}6 \text { renal, } 1 \\
\text { perirenal }\end{array}$ & $2.2-10.1$ & Nephrectomy & BEN & NA & NA & $\begin{array}{l}\text { POS } \\
\text { in } 6\end{array}$ & $\begin{array}{l}\text { Pierson DM et al., Mod Pathol } \\
18: 159\end{array}$ \\
\hline 36 & 2005 & 51 & F & Flank pain & NA & $\begin{array}{l}\text { Renal } \\
\text { capsule }\end{array}$ & 10 & Nephrectomy & BEN & NA & NA & POS & Yamaguchi T, Urology 65:175 \\
\hline 37 & 2005 & 51 & $\mathrm{~F}$ & Fever elevation & $\mathrm{R}$ & $\begin{array}{l}\text { Renal } \\
\text { capsule }\end{array}$ & 13 & Nephrectomy & BEN & NA & NA & $\begin{array}{l}\text { POS } \\
\text { (focal) }\end{array}$ & $\begin{array}{l}\text { Jhonson TR et al., J Comput } \\
\text { Assist Tomogr 29:481 }\end{array}$ \\
\hline 38 & 2005 & 83 & $\mathrm{~F}$ & Incidental & $\mathrm{L}$ & Kidney & 11 & Nephrectomy & BEN & NA & NA & POS & $\begin{array}{l}\text { Kawagoe M, Nishinihon J Urol } \\
67: 568\end{array}$ \\
\hline 39 & 2006 & 76 & $\mathrm{M}$ & Incidental & $\mathrm{L}$ & Kidney & 12 & Nephrectomy & MAL & 4 & $\begin{array}{l}\text { Lung } \\
\text { metastasis }\end{array}$ & $\begin{array}{l}\text { POS (be- } \\
\text { nign site) }\end{array}$ & $\begin{array}{l}\text { Fine SW et al., Arch Pathol Lab } \\
\text { Med 130:857 }\end{array}$ \\
\hline 40 & 2006 & 18 & F & Flank pain & $\mathrm{L}$ & Kidney & 3 & Nephrectomy & BEN & 15 & NED & POS & $\begin{array}{l}\text { Koroku M et al., Hinyokika } \\
\text { Kiyo 52:705 }\end{array}$ \\
\hline 41 & 2006 & 4 & M & NA & $\mathrm{R}$ & Kidney & 8 & Nephrectomy & BEN & NA & NA & NA & $\begin{array}{l}\text { Provance et al., Clin Pediatr } \\
45: 871\end{array}$ \\
\hline 42 & 2006 & 85 & $\mathrm{M}$ & Flank pain & $\mathrm{L}$ & Kidney & 4.5 & Nephrectomy & BEN & NA & NA & POS & $\begin{array}{l}\text { Kohl SK et al., Arch Pathol Lab } \\
\text { Med 130:117 }\end{array}$ \\
\hline 43 & 2006 & 54 & $\mathrm{M}$ & Incidental & $\mathrm{R}$ & Kidney & NA & Nephrectomy & BEN & 16 & NED & POS & $\begin{array}{l}\text { Tanaka M et al., Hinyokika } \\
\text { Kiyo 52:79 }\end{array}$ \\
\hline 44 & 2006 & 36 & $\mathrm{M}$ & Flank pain & $\mathrm{R}$ & Kidney & NA & Nephrectomy & BEN & NA & NA & NA & $\begin{array}{l}\text { Alvarez Mugica M et al., Arch } \\
\text { Esp Urol 59:195 }\end{array}$ \\
\hline
\end{tabular}




\begin{tabular}{|c|c|c|c|c|c|c|c|c|c|c|c|c|c|}
\hline 45 & 2007 & 26 & M & Incidental & $\mathrm{R}$ & Kidney & 7 & Nephrectomy & BEN & 6 & NED & POS & $\begin{array}{l}\text { Constantinidis C et al., The Can } \\
\text { J Urol 14:3583 }\end{array}$ \\
\hline 46 & 2007 & 70 & M & $\begin{array}{l}\text { Flank pain and } \\
\text { macrohematuria }\end{array}$ & $\mathrm{L}$ & Kidney & 15 & Nephrectomy & BEN & 6 & NED & POS & $\begin{array}{l}\text { Znati K et al., Reviews in Urol } \\
\text { 9:36 }\end{array}$ \\
\hline 47 & 2007 & 51 & $\mathrm{~F}$ & Flank pain & $\mathrm{L}$ & Kidney & 4 & Nephrectomy & BEN & 10 & NED & POS & $\begin{array}{l}\text { Bozkurt SU et al., APMIS } \\
115: 259\end{array}$ \\
\hline 48 & 2007 & 66 & $\mathrm{~F}$ & $\begin{array}{l}\text { Abdominal mass and } \\
\text { macrohematuria }\end{array}$ & & Kidney & 11 & Nephrectomy & BEN & NA & NA & NA & $\begin{array}{l}\text { Kakoi N et al., Japn J Urol Surg } \\
20 \text { supple } 598\end{array}$ \\
\hline 49 & 2007 & $60 s$ & M & Incidental & $\mathrm{R}$ & Kidney & 3 & Nephrectomy & BEN & 3 & NED & NA & $\begin{array}{l}\text { Yoshida T et al., Hinyokika } \\
\text { Kiyo 53:745 }\end{array}$ \\
\hline 50 & 2008 & 34 & $\mathrm{~F}$ & Flank pain & $\mathrm{L}$ & Kidney & 9 & Nephrectomy & MAL & 21 & NED & POS & $\begin{array}{l}\text { Magro G et al., APMIS } \\
115: 1020\end{array}$ \\
\hline 51 & 2008 & 67 & M & Macrohematuria & $\mathrm{L}$ & Kidney & 7 & Nephrectomy & BEN & 10 & NED & POS & $\begin{array}{l}\text { Amano T et al., Hinyokika Kiyo } \\
54: 357\end{array}$ \\
\hline 52 & 2008 & 44 & F & Incidental & $\mathrm{L}$ & Kidney & 5.8 & Nephrectomy & BEN & 40 & NED & POS & $\begin{array}{l}\text { Hirabayashi J et al., Hinyokika } \\
\text { Kiyo } 54: 357\end{array}$ \\
\hline 53 & 2009 & 75 & $\mathrm{~F}$ & Incidental & $\mathrm{L}$ & Kidney & 4.5 & Nephrectomy & BEN & 9 & NED & POS & $\begin{array}{l}\text { Hirano D et al., Mod Mol } \\
\text { Morphol 42:239 }\end{array}$ \\
\hline 54 & 2009 & 64 & $\mathrm{~F}$ & Cough & $\mathrm{L}$ & Kidney & 2.5 & Biopsy & BEN & 12 & NED & POS & $\begin{array}{l}\text { Petrella F et al., Minerca Chir } \\
64: 669\end{array}$ \\
\hline 55 & 2009 & 35 & M & Incidental & $\mathrm{R}$ & Kidney & 8 & $\begin{array}{l}\text { Partial } \\
\text { nephrectomy }\end{array}$ & BEN & NA & NA & POS & $\begin{array}{l}\text { Makris A et al., Can J Urol } \\
16: 4854\end{array}$ \\
\hline 56 & 2009 & 72 & F & Abdominal mass & $\mathrm{L}$ & Kidney & 19 & Nephrectomy & MAL & NA & NA & NA & Marzi M et al., Urologia 76:112 \\
\hline 57 & 2009 & 76 & $\mathrm{~F}$ & Incidental & $\mathrm{R}$ & Kidney & 2.5 & Nephrectomy & BEN & 48 & NED & POS & $\begin{array}{l}\text { Yoneyama T et al., Hinyokika } \\
\text { Kiyo 55:479 }\end{array}$ \\
\hline 58 & 2009 & 50 & M & Incidental & $\mathrm{L}$ & Kidney & 5.5 & Nephrectomy & BEN & NA & NED & POS & $\begin{array}{l}\text { Matsumoto T et al., Japn J Urol } \\
\text { Surg 22:230 }\end{array}$ \\
\hline 59 & 2009 & 63 & M & Incidental & $\mathrm{L}$ & Kidney & 5.3 & Nephrectomy & MAL & NA & NA & POS & $\begin{array}{l}\text { Murayama S et al., Japn J Urol } \\
\text { Surg 22:230 }\end{array}$ \\
\hline 60 & 2009 & 51 & $\mathrm{~F}$ & Incidental & $\mathrm{R}$ & Kidney & 12 & Nephrectomy & BEN & NA & NA & POS & $\begin{array}{l}\text { Ogushi S et al., Japn J Urol Surg } \\
22: 230\end{array}$ \\
\hline 61 & 2009 & 75 & M & NA & $\mathrm{L}$ & Kidney & 3 & $\begin{array}{l}\text { Nephroureterec- } \\
\text { tomy }\end{array}$ & BEN & NA & NA & POS & $\begin{array}{l}\text { Kobori Y et al., Hinyokika Kiyo } \\
\text { 55:305 }\end{array}$ \\
\hline 62 & 2010 & 39 & M & Dysuria & $\mathrm{L}$ & Kidney & 25 & Nephrectomy & BEN & 12 & NED & POS & $\begin{array}{l}\text { Taza L et al., Actas Urol Esp } \\
\text { 34:568 }\end{array}$ \\
\hline 63 & 2010 & 39 & $\mathrm{~F}$ & Abdominal fullness & $\mathrm{L}$ & Kidney & 20 & $\begin{array}{l}\text { Embolization and } \\
\text { nephrectomy }\end{array}$ & BEN & 6 & NED & POS & $\begin{array}{l}\text { Yamaguchi Y et al., Hinyokika } \\
\text { Kiyo 56:435 }\end{array}$ \\
\hline 64 & 2011 & 44 & M & Macrohematuria & $\mathrm{L}$ & Kidney & NA & $\begin{array}{l}\text { Embolization and } \\
\text { nephrectomy }\end{array}$ & BEN & NA & NA & NA & $\begin{array}{l}\text { Saegusa M et al., Nishinihon J } \\
\text { Urol 68:187 }\end{array}$ \\
\hline 65 & 2011 & 52 & $\mathrm{~F}$ & Abdominal pain & $\mathrm{R}$ & Kidney & 18 & $\begin{array}{l}\text { Nephrectomy and } \\
\text { thrombectomy }\end{array}$ & BEN & 6 & NED & POS & $\begin{array}{l}\text { Naveen HN et al., Urol Ann } \\
3: 158\end{array}$ \\
\hline 66 & 2011 & 72 & F & Abdominal mass & $\mathrm{L}$ & Kidney & 19 & Nephrectomy & MAL & 15 & NED & $\begin{array}{l}\text { POS } \\
\text { (focal) }\end{array}$ & $\begin{array}{l}\text { Marzi M et al., Minerva Urol } \\
\text { Nephrol 63:109 }\end{array}$ \\
\hline 67 & 2011 & 50 & $\mathrm{~F}$ & Flank pain & $\mathrm{R}$ & Kidney & 15 & Nephrectomy & MAL & 30 & NED & POS & $\begin{array}{l}\text { Tsan-Yu Hsieh, Diag Pathol } \\
6: 96\end{array}$ \\
\hline $\begin{array}{l}\text { Our } \\
\text { case }\end{array}$ & & 48 & $\mathrm{M}$ & Abdominal mass & $\mathrm{R}$ & Kidney & 29 & Nephrectomy & BEN & 107 & NED & $\begin{array}{l}\text { POS } \\
(55 \%)\end{array}$ & \\
\hline
\end{tabular}

$\mathrm{M}=$ Male; $\mathrm{F}$ = female; $\mathrm{NA}=$ not available; $\mathrm{R}=$ right; $\mathrm{L}=$ left; $\mathrm{BE}=$ benign; $\mathrm{MAL}=$ malignant; $\mathrm{DNOD}=$ died not of disease; $\mathrm{NED}=$ no evidence of disease; $\mathrm{POS}=$ positive. * CD34 immunoreactivity (the extent of positive area is shown in parentheses, if information is available). 


\section{Case Reports in \\ Nephrology and Urology}

\begin{tabular}{l|l}
\hline Case Rep Nephrol Urol 2013;3:1-8 \\
\hline DOI: $\underline{10.1159 / 000346850}$ & $\begin{array}{l}\text { ○ 2013 S. Karger AG, Basel } \\
\text { www.karger.com/cru }\end{array}$ \\
\hline
\end{tabular}

Sasaki et al.: Distant Metastasis from Benign Solitary Fibrous Tumor of the Kidney

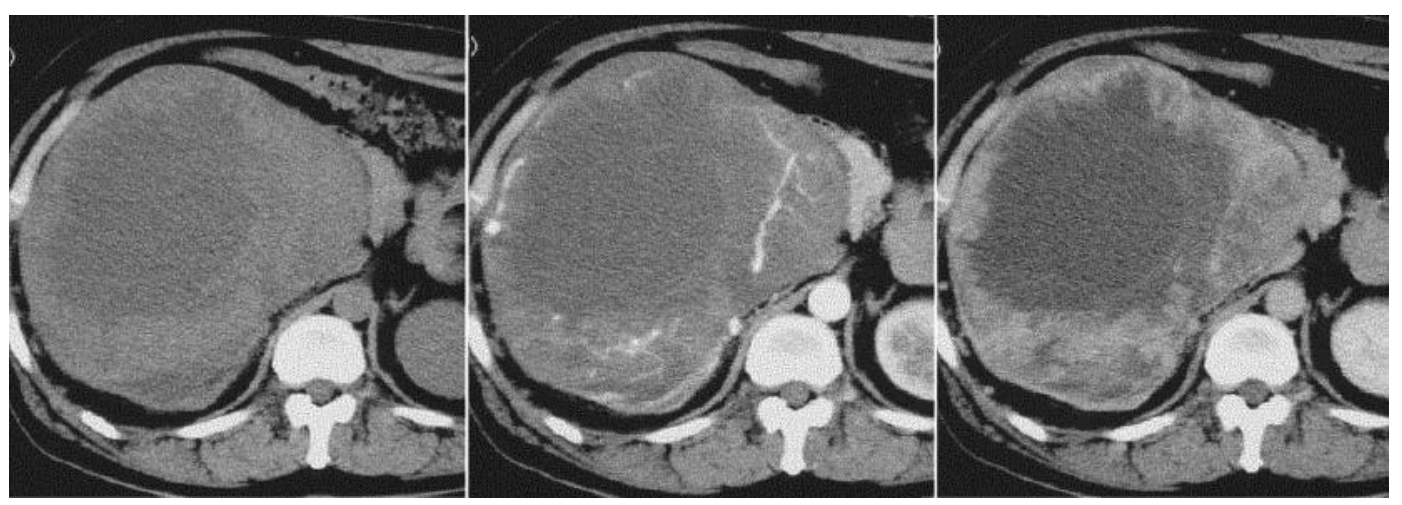

Fig. 1. CT findings. A large right renal tumor was observed on plain CT (left). Blood vessels were enhanced in the early phase (middle), and the tumor was enhanced in the delayed phase (right).

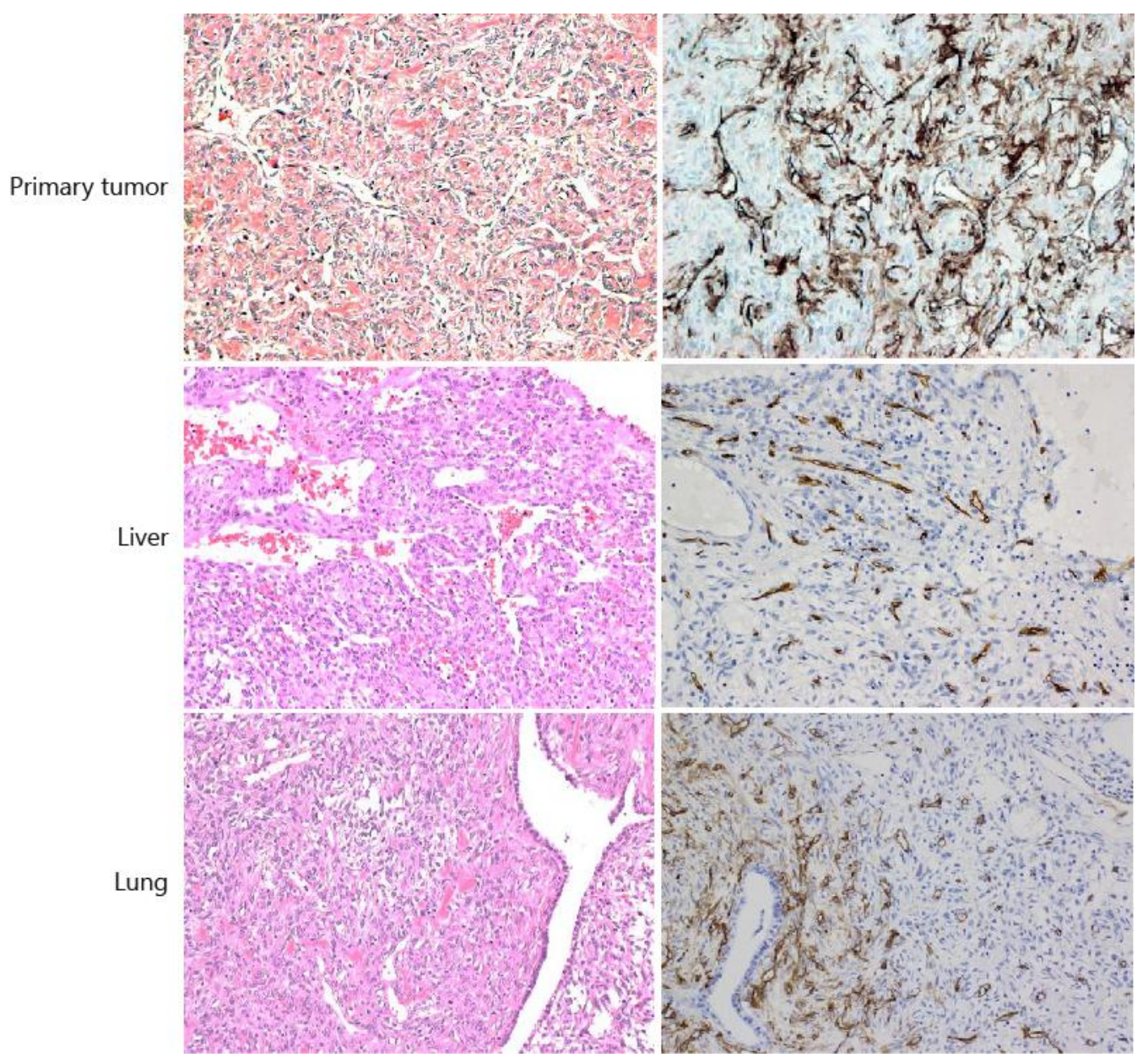

Fig. 2. Microscopic features of the solitary fibrous tumor. The primary renal tumor displayed a hemangiopericytomatous growth pattern (upper left). Immunohistochemical staining of CD34 was positive in $55 \%$ of the primary tumor (upper right). Resected tissue from the liver (middle left) and lungs (lower left). CD34 expression was completely absent from the resected liver tumor (middle right) but weakly positive in the resected lung tumor (lower right). 
Case Reports in

Nephrology and

Urology
Case Rep Nephrol Urol 2013;3:1-8

DOI: $\underline{10.1159 / 000346850}$

Sasaki et al.: Distant Metastasis from Benign Solitary Fibrous Tumor of the Kidney
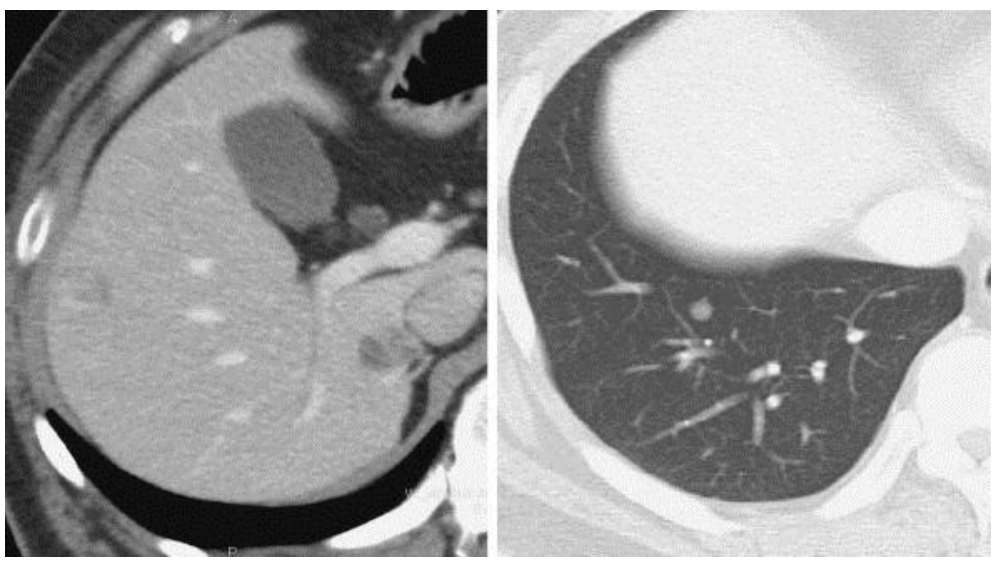

Fig. 3. Follow-up computed tomography revealed liver metastasis and lung metastasis. 